\title{
Transmission of vibration through gloves: effects of contact area
}

\begin{abstract}
For three samples of material $(12.5,25.0$ and $37.5 \mathrm{~mm}$ diameter) from each of three gloves, the dynamic stiffnesses and the vibration transmissibilities of the materials (to both the palm of the hand and the thenar eminence) were measured at frequencies from 10 to $300 \mathrm{~Hz}$. Additional measurements showed the apparent masses of the hand at the palm and the thenar eminence were independent of contact area at frequencies less than about $40 \mathrm{~Hz}$, but increased with increasing area at higher frequencies. The stiffness and damping of the glove materials increased with increasing area. These changes caused material transmissibilities to the hand to increase with increasing area. It is concluded that the size of the area of contact has a large influence on the transmission of vibration through a glove to the hand. The area of contact should be well-defined and controlled when evaluating the transmission of vibration through gloves.
\end{abstract}

Keyword: Anti-vibration gloves; Biodynamics; Hands; Impedance; Transmissibility 